\title{
Minimizing Execution Costs when using Globally Distributed Cloud Services
}

\author{
Suraj Pandey, Adam Barker, Kapil Kumar Gupta, Rajkumar Buyya \\ Cloud Computing and Distributed Systems Laboratory \\ Department of Computer Science and Software Engineering \\ The University of Melbourne, Australia \\ \{spandey, adbarker, kgupta, raj\}@csse.unimelb.edu.au
}

\begin{abstract}
Cloud computing is an emerging technology that allows users to utilize on-demand computation, storage, data and services from around the world. However, Cloud service providers charge users for these services. Specifically, to access data from their globally distributed storage edge servers, providers charge users depending on the user's location and the amount of data transferred. When deploying data-intensive applications in a Cloud computing environment, optimizing the cost of transferring data to and from these edge servers is a priority, as data play the dominant role in the application's execution. In this paper, we formulate a non-linear programming model to minimize the data retrieval and execution cost of data-intensive workflows in Clouds. Our model retrieves data from Cloud storage resources such that the amount of data transferred is inversely proportional to the communication cost. We take an example of an 'intrusion detection' application workflow, where the data logs are made available from globally distributed Cloud storage servers. We construct the application as a workflow and experiment with Cloud based storage and compute resources. We compare the cost of multiple executions of the workflow given by a solution of our non-linear program against that given by Amazon CloudFront's 'nearest' single data source selection. Our results show a savings of three-quarters of total cost using our model.
\end{abstract}

\section{Introduction}

Scientific and commercial applications are leveraging the power of distributed computing and storage resources $[5,19]$. These resources are available either as part of general purpose computing infrastructure such as Clusters and Grids, or through commercially hosted services such as Clouds [1]. Clouds have been defined to be a type of parallel and distributed system consisting of inter-connected and virtualized computers. These computers can be dynamically provisioned as per users' requirements [4]. Thus, to achieve better performance and scalability, applications could be managed using commercial services provided by Clouds, such as Amazon AWS, Google AppEngine, and Microsoft Azure. Some of these cloud service providers also have data distribution services, such as Amazon CloudFront ${ }^{1}$. However, the cost of computing, storage and communication over these resources could be very high for compute-intensive and data-intensive applications .

Data mining is an example application domain that comprises of data-intensive applications often with large distributed data and compute-intensive tasks. The data to be mined may be widely distributed depending on the nature of the application. As the size of these data-sets increases over time, the analysis of distributed data-sets on computing resources by multiple users (repeated executions) has the following challenges:

- A well-designed application workflow: Large number of data-sets and mining tasks make the application complex.

- Minimization of communication and storage costs: Large size and number of distributed data-sets make the application data-intensive.

- Minimization of repeated data mining costs: Cost of computing (classification/knowledge discovery) and transferring of data increases as the number of iterations/data-sets increase.

In this paper, we address the challenges listed above for data-intensive workflows by making the following three contributions:

1. We take Intrusion detection as a data mining application which will be referenced throughout the remainder of this paper. This application has all the features as listed in the previous paragraph when executing commercially [19]. We design the application as a workflow that simplifies the basic steps of data mining into blocks.

2. We model the cost of execution of an intrusion detection workflow on Cloud resources using a Non-Linear Programming (NLP) model. The NLP-model retrieves

${ }^{1}$ http://aws.amazon.com/cloudfront/ 
data partially from multiple data sources based on the cost of transferring data from those sources to a compute resource, so that the total cost of data-transfer and computation cost on that compute resource is minimized.

3. We then apply the NLP-model on the intrusion detection application to minimize repeated execution costs when using commercial compute and storage resources. As an example, we compare the costs between our model and Amazon CloudFront.

The remainder of the paper is organized as follows: we present an intrusion detection application and its workflow design in Section 2; cost minimization problem using NLP model in Section 3; the NLP-model and its use for the intrusion detection application in Section 4; experimental setup in Section 5 and analysis in Section 6; related work in Section 7. Finally, we conclude the paper in Section 8.

\section{Intrusion Detection Using Data from Dis- tributed Data Sources}

First, we describe a use-case for Internet worm detection. Then, we describe the process of intrusion detection in general and present a workflow design for executing data mining steps over distributed intrusion data logs.

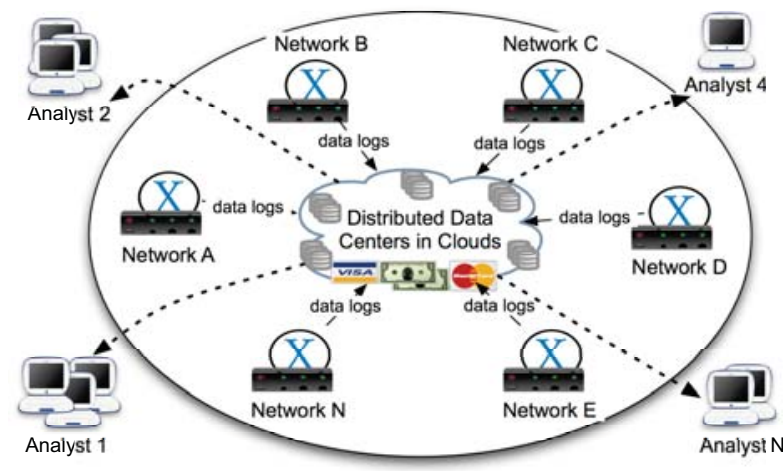

Figure 1: Global Intrusion Detection scenario

\subsection{Intrusion detection scenario}

Intrusion detection as defined by the SysAdmin, Audit, Networking, and Security $\left(\mathrm{SANS}^{2}\right)$ institute is the act of detecting actions that attempt to compromise the confidentiality, integrity or availability of a resource. We take an example of detecting the spread of a malicious worm over the Internet.

In practice, a large number of independent networks spanning throughout the Internet share their network logs to detect such an outbreak. The logs from each individual network are continuously fed to the Amazon Cloud storage (or some other services), which distributes them to globally distributed edge servers.

\footnotetext{
${ }^{2}$ http://www.sans.org/resources/idfaq/what isid.php
}

The aim of the intrusion detection system (or the analyst) is to analyze these combined logs to detect an outbreak of a worm. Such analysts can be located at multiple locations close to some of the data sources but at a large network distance from a majority of the other data sources.

Assuming that every intrusion detection system (or analyst) follows the same data mining process, which we describe later in the paper, the Naive approach is to separately aggregate the log data from all independent networks for every analyst. It is not hard to visualize the redundancy in the data transfer (for each individual network) and hence the cost associated with such massive amount of data transfers.

Using the distributed edge servers, we can minimize the cost of data transfer to each individual intrusion detection system (analyst). We represent this scenario in Figure 1. With an aim to minimize the cost of data transfer, we develop a non linear programming based approach, described later in the paper, and compare it with the standard nearest source approach adopted by CloudFront and observe that our model achieves a significant savings of three-quarters of the total cost.

\subsection{Intrusion detection process as a workflow}

Data mining techniques have become prominent in detecting intrusions $[10,9]$. Detecting intrusions can be considered as finding the outliers (or unusual activities) and, hence, data mining can be easily applied to perform this task.

We modeled the intrusion detection process as a workflow as illustrated by Figure 2. The figure separates the training, testing, and real-time processes into blocks as Block A, Block B and Block C, respectively. The first step for training is to collect some training data, which can be the contents of IP packets, web server logs, etc.. Collected data are pre-processed (normalization, adding missing values, etc), represented in a format that is supported by the data mining tool (in our case it is .arff format), and pruned to contain a small set of features that are significant to improve the performance and accuracy of the system. The feature selection is the attribute selection (AS) in the figure. Applying these selected features on the training data is the Filter $(\mathrm{F})$ process. Finally, with the reduced training data, we apply different algorithms to train corresponding models. In our experiments, we selected well known methods for data mining and intrusion detection such as Naive Bayes (NB), Decision Trees (DT) and Support Vector Machines (SMO).

To evaluate the effectiveness of the trained models, we perform the testing on the test data (Block B in the figure). We repeat the same steps as in Block A on the test data, except the AS. We then use the trained model to generate output using the test data. Finally, we select the best performing model based on the accuracy of classification of 


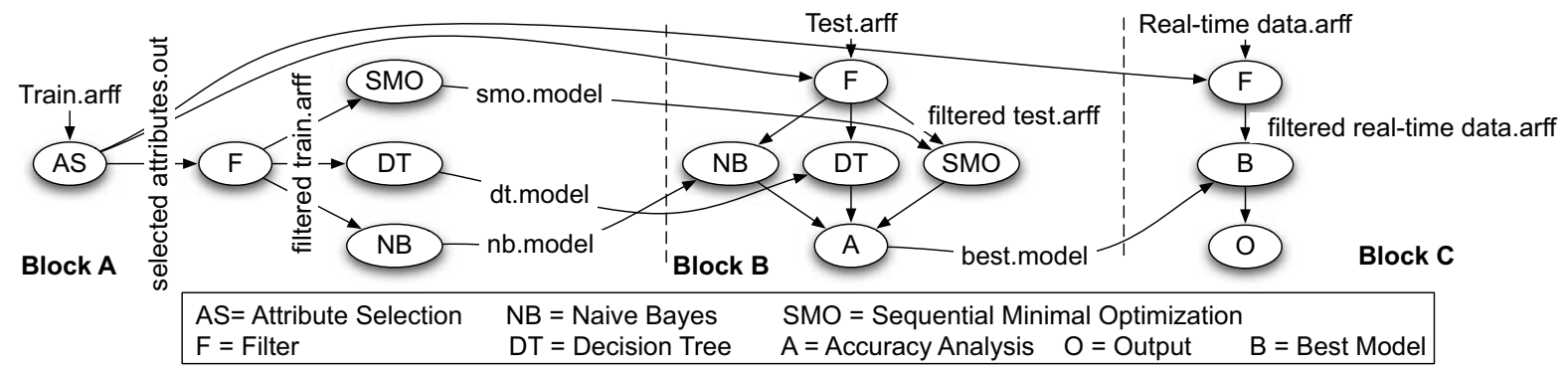

Figure 2: Intrusion Detection workflow

individual models, denoted as (A) in the figure. This model, which is the most accurate, is then used on the real-time data from distributed data sources (Block $\mathrm{C}$ in the figure). Realtime data is filtered (F), then the best model applied (B) and the output $(\mathrm{O})$ is generated.

The input data for Block A are the training data, Block $\mathrm{B}$ are the testing data and Block $\mathrm{C}$ are the real-time data. The output data from each task are labels. At the end of Block A's execution, three models (nb.model, dt.model \& smo.model) files are created, which then becomes input for Block B. Block B's execution generates of the most accurate model (best.model) as input for Block C. Block $\mathrm{C}$ then applies the model for the real-time data logs to obtain the intrusion classification.

\section{Cost Minimization using Non-Linear Pro- gramming Model}

\subsection{Notations and problem}

We denote an application workflow using a Directed Acyclic Graph (DAG) by $G=(V, E)$, where $V=\left\{T_{1}, \ldots, T_{n}\right\}$ is the set of tasks, and $E$ represents the data dependencies between these tasks, that is, $t_{d a t a}{ }^{k}=\left(T_{j}, T_{k}\right) \in E$ is the data produced by $T_{j}$ and consumed by $T_{k}$.

We have a set of storage sites $S=\{1, \ldots, i\}$, a set of compute sites $P=\{1, \ldots, j\}$, and a set of tasks $T=$ $\{1, \ldots, k\}$. We assume the 'average' computation time of a task $T_{k}$ on a compute resource $P_{j}$ for a certain size of input is known. Then, the cost of computation of a task on a compute host is inversely proportional to the time it takes for computation on that resource. We also assume the cost of unit data access $t_{x c o s} t_{i, j}$ from a storage resource $S_{i}$ to a compute resource $P_{j}$ is known. The transfer cost is fixed by the service provider (e.g. Amazon CloudFront) or can be calculated according to the bandwidth between the sites. We assume that these costs are non-negative, symmetric, and satisfy the triangle inequality: that is, txcost $_{i, j}=$ txcost $_{j, i}$ for all $i, j \in N$, and txcost $_{i, j}+$ txcost $_{j, k} \geq$ txcost $_{i, k}$ for all $i, j, k \in N$. These relations can be expressed as: ecost $\propto 1 /$ \{execution time or capability of resource $\}$ txcost $\propto$ bandwidth $O R=($ tx cost $/$ unit data $) /$ site total cost of computation:

$$
C \leq \text { ecost } * \text { etime }+ \text { txcost } * \text { data }+ \text { overheads }
$$

The cost-optimization problem is: Find a feasible set of 'partial' data-sets $\left\{d_{i, j}^{k}\right\}$ that must be transferred from storage host $S_{i}$ to compute host $P_{j}$ for each task $\left(T_{k} \in V\right)$ such that the total retrieval cost and computation cost of the task on $P_{j}$ is minimal, for all the tasks in the workflow (not violating dependencies).

\subsection{Non-linear model}

Here, we try to get the minimum cost by formulating a non-linear program for the cost-optimization problem, as depicted in Figure 3. The formulation uses two variables $y, d$ and pre-computed values $t x c o s t$, ecost, txtime, etime as listed below:

- $y$ characterizes where each task is processed. $y_{j}^{k}=1$ iff task $T_{k}$ is processed on processor $P_{j}$.

- $d$ characterizes the amount of data to be transferred to a site. e.g. $d_{i, j}^{k}=50.2$ denotes 50.2 units of data are to be transferred from $S_{i} \Rightarrow P_{j}$ for task $T_{k}$

- txcost characterizes the cost of data transfer for a link per data unit. e.g. $t x \operatorname{cost}_{i, j}=10$ denotes the cost of data transfer from $S_{i} \Rightarrow P_{j}$. It is added to the overall cost iff $d_{i, j}^{k}>0 \& y_{j}^{k}=1$.

- ecost characterizes the cost of computation (usage time) of a processor. e.g. ecost ${ }_{j}=1$ denotes the cost of using a processor $P_{j}$. It is added to the overall cost iff $y_{j}^{k}=1$.

- txtime characterizes the average time for transferring unit data between two sites. e.g. txtime $_{i, j}=50 \mathrm{de}$ notes the time for transferring unit data from $S_{i} \Rightarrow P_{j}$. It is added to the Execution Time (ET) for every task iff $d_{i, j}^{k}>0 \& y_{j}^{k}=1$.

- etime characterizes the computation time of a task averaged over a set of known and dedicated resources. e.g. etime ${ }_{j}^{k}=20$ denotes the time for executing a task $T_{k}$ on a processor $P_{j}$. It is added to ET iff $y_{j}^{k}=1$.

The constraints can be described as follows: 


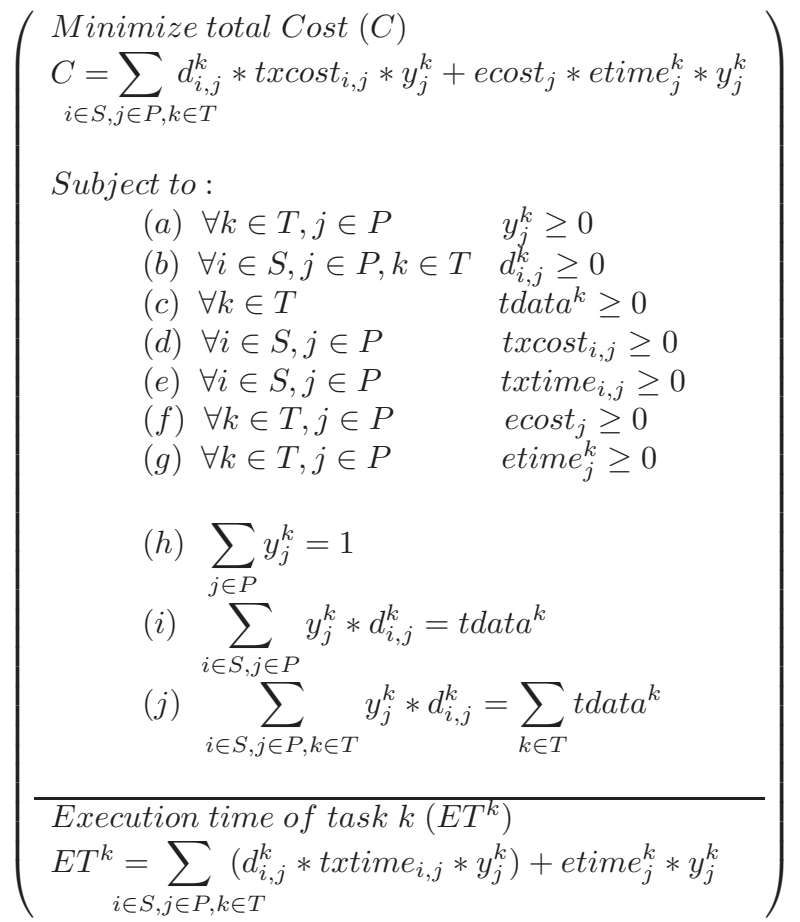

Figure 3: NLP-model

- (a) \& (h) ensure that each task $k \in T$ is computed only once at processor $j \in P$ when the variable $y_{j}^{k}>0$. For partial values of $y_{j}^{k}$, we round up/down to the nearest integer $(0$ or 1$)$. Tasks are not partitioned or migrated.

- (b) \& (c) ensure that partial data transferred and total data required by a task cannot be negative.

- (d), (e), (f) and (g) ensure that cost and time values are all positive.

- (i), (a) \& (b) ensure that partial-data are transferred only to the resource where a task is executed. For all such transfers, the sum of data transferred should equal to the data required by the task, which is $t$ data ${ }^{k}$.

- (j) ensures that the total data transfer for all the tasks are bounded by the sum of data required by each task. This is important for the solvers to relate (h), (i) \& (j),

- (i) \& (j) combined ensure that whenever partial-data $d_{i, j}^{k}$ is transferred to a compute host $P_{j}$, then a compute host must have been selected at $j\left(y_{j}^{k}=1\right)$, and that total data transfer never exceeds the bound $t d a t a^{k}$ for each task and in total.

To get an absolute minimum cost, we map the tasks in the workflow onto resources based only on cost optimization (not time). This eliminates the time dependencies between tasks. However, the task to compute-resource mappings and data-source to compute-resource mappings minimizes the cost of execution but not the makespan. The execution time of a task $\left(E T^{k}\right)$ is calculated based on the cost-minimized mappings given by the solver. The total: $\sum_{k \in T}\left(E T^{k}+\right.$ waiting_time $)$ is the makespan of the workflow with the minimum cost, where the waiting time denotes the minimum time a task has to wait before its parents finish execution.

\section{Cost Minimization for The Intrusion De- tection Application}

In this section, we describe the method we used to solve the non-linear program formulated in Section 3. We then describe how we applied the solution for minimizing the total cost of execution to the intrusion detection application workflow.

NLP-solver: We wrote a program using the Modelling Language for Mathematical Programming (AMPL) [7] for solving our NLP-model. We used DONLP2[15], a nonlinear program solver, to solve the model. The computation time of the solver to reach a solution (for a maximum of 2000 iterations) was less than 2 seconds, which is insignificant as compared to the data-transfer time in our experiments.

Partial-data retrieval and task-to-resource mapping: Based on the integer values of $y_{j}^{k}$ given by DONLP2, we statically mapped the tasks in the intrusion detection application workflow to each compute resource $P_{j}$. Data retrievals are also fixed for each ready task from each $S$ based on the value of $d_{i, j}^{k}$ and $y_{j}^{k}=1$. The steps of mapping and data retrievals are given in Algorithm 1. The heuristic computes the values for task mapping $y_{j}^{k}$ and $d_{i, j}^{k}$ for all the tasks in the beginning according to the solution given by a NLP-solver. As all the tasks in the workflow are mapped initially, the for loop preserves the dependencies of the tasks by dispatching only the ready tasks to the resources. For dispatched tasks, partial data retrievals to the assigned compute resource occur from chosen resources. All child tasks wait for their parents to complete, after which they appear in the ready list for dispatching. The scheduling cycle completes after all the tasks are dispatched successfully. The output data of each completed task is staged back to the Cloud storage as part of the task's execution. The Cloud storage should ensure that the files are distributed to the edge-servers within certain time bound such that child tasks do not have to wait for availability of data longer than downloading directly from the Cloud's central server.

\section{Experimental Setup}

In this Section, we describe Intrusion Detection data and tools, the experimental setup, and the results. 


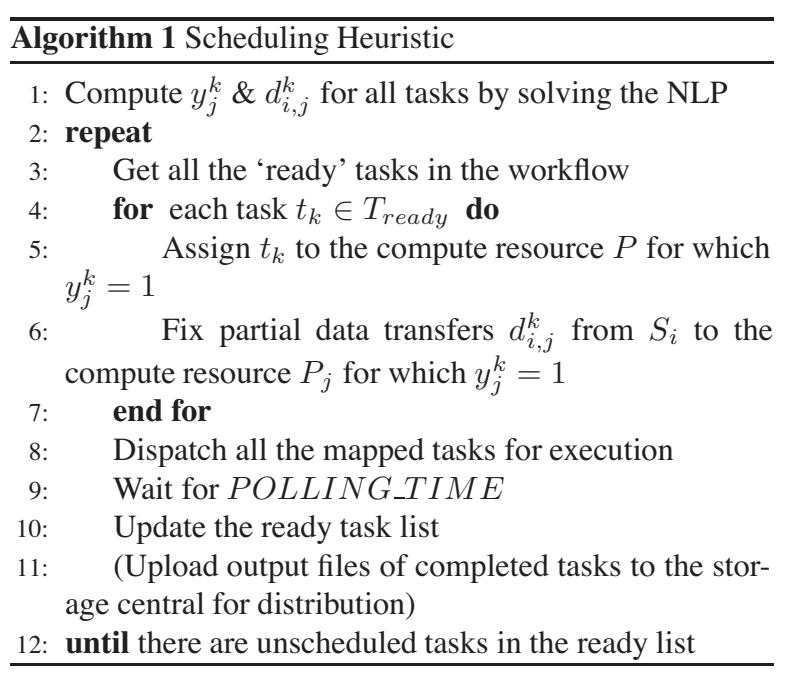

\subsection{Intrusion detection application data and tools}

Data: For our experiments, we used part of the benchmark KDD'99 intrusion data set ${ }^{3}$. This database contains a standard set of data to be audited, which includes a wide variety of intrusions simulated in a military network environment. We use 10 percent of the total training data and 10 percent of the test data (with corrected labels), which are provided separately. Each record in the data set represents a connection between two IP addresses, starting and ending at defined times and protocol. Furthermore, every record is represented by 41 different features. Each record represents a separate connection and is hence considered to be independent of any other record. Training data are either labeled as normal or as one of the 24 different types of attack. These 24 attacks can be grouped into four classes; Probing, Denial of Service (DoS), unauthorized access from a remote machine (R2L) and unauthorized access to root (U2R). Similarly, test data are also labeled as either normal or as one of the attacks belonging to the four attack groups.

To perform data mining we used algorithms implemented in an open source WEKA library [17]. We used three types of probabilistic classification models: Naive Bayes, decision tree and Sequential Minimal Optimization (SMO), from the WEKA library. The number of log-data analysis for detecting intrusion varies depending on the characteristics of the log data. To reflect all types of scenarios, we perform the real-time log-data analysis for 10 times. We interpolate the cost for 10,000 times execution by multiplying the cost of 10 executions multiplied by 1000 .

The total data used by the intrusion detection workflow (Figure 2) is divided into $30 \mathrm{MB}, 60 \mathrm{MB}, 90 \mathrm{MB}$ and $120 \mathrm{MB}$. This was be achieved by filtering the training, testing and real-time data by random sampling.

\footnotetext{
${ }^{3}$ http://kdd.ics.uci.edu/databases/kddcup99/kddcup99.html.
}

\subsection{Middleware and tools}

We used Workflow Engine [14] for scheduling and managing workflow executions on Cloud resources. The engine together with the application broker are part of the Cloudbus Toolkit [3]. We use the scheduling heuristic listed in Algorithm 1. Both, multi-site partial downloads and CloudFront downloads were carried out over HTTP using JPartialDownloader tool ${ }^{4}$. HTTP/1.1 range requests allow a client to request portions of a resource.

\subsection{Distributed compute and storage resources}

For selecting the nearest storage location of a file relative to a compute resource, we use the functionality of Amazon CloudFront. CloudFront fetches data to a compute resource from the nearest edge-server. The data transfer cost (per GB) from the edge locations is presented in Table 1. The data transfer cost (DTx cost) from the CloudFront to the execution sites is based on the edge location through which the content is served. We assume the data transfer cost to and from a storage location to be equal in all our experiments. This simplifies the model for the selection of storage sites for partial data retrievals and data upload. For partial data retrievals, all the resources listed in Table 1 also served as storage resources. For our experiments, we ignored the data storage cost on Clouds, which could easily be added to the overall execution cost as a constant (e.g. $\$ 0.150$ per GB for the first $50 \mathrm{~TB} /$ month of storage used $^{5}$ ).

We used compute resources from US, Europe and Asia as listed in Table 1. The execution cost (Ex cost) on each $\mathrm{CPU}$ is calculated based on the number of cores (cost is similar to Amazon EC2 instances) available.

\section{Analysis}

We now present results obtained by executing the intrusion detection application workflow using globally distributed resources as listed in Table 1.

\subsection{Experiment objectives}

We conduct the following two classes of experiments:

1. Measure total cost when using commercial Cloud as content distribution and publicly available compute resources for execution $\left(\right.$ ecost $_{j}=0$, txcost $\left._{i, j}>0\right)$.

2. Measure total cost of execution when using commercial Cloud for content storage, distribution and execution $\left(e_{c}\right.$ ost $\left.>0, t x \cos t_{i, j}>0\right)$

The first experiment (subsection 6.2.1) measures the cost of data transfer if Cloud resources were used only for data distribution and tasks executed on publicly available compute resources. In this scenario, the compute resources in Table 1 served both as storage (mimicking distributed Cloud storage) and compute resources. We use a solution

\footnotetext{
${ }^{4}$ http://jpd.sourceforge.net/

${ }^{5}$ http://aws.amazon.com/s3/\#pricing
} 
to our model for determining quantity of partial data transfers from the distributed storage such that the transfer cost is minimized. The tasks are mapped to the compute resources such that the partial transfers have minimum cost.

The second experiment (subsection 6.2.2) measures the cost of executing the application on Cloud resources, with non-zero data transfer and computation costs. In this scenario, our model gives a solution for minimizing both partial data transfers and computation costs, with tasks mapped to resources accordingly. Here too, the compute-servers in Table 1 serve as distributed Cloud storage and compute resources.

We compare the costs obtained from each of the above experiments against the cost incurred when using datatransfers from nearest (with respect to the compute resource where the task is assigned) Cloud storage resource. We measure the total cost incurred for transferring data from nearest location by making compute-resource cost: zero (relating to publicly available resources) and non-zero (relating to commercial Cloud resources), consecutively.

We finally compare the cost savings when using NLP based task+data resource selection against CloudFront's data resource selection.

\subsection{Results}

The results obtained are an average of 15 executions. The cost values in Figures 4 and 5 are for executing a single instance of the intrusion detection workflow. The cost values in Figure 6 are the result of executing the workflow 10,000 times (the cost of 10 executions multiplied by 1000).

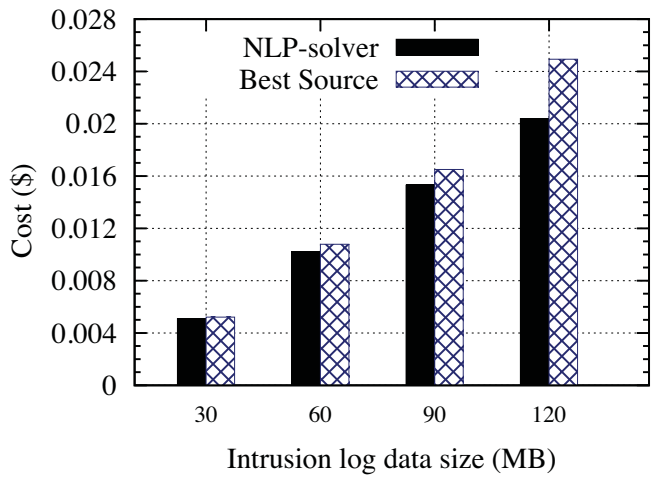

Figure 4: Comparison of transfer cost with no execution cost.

\subsubsection{Scenario 1: Data in Cloud and execution on pub- lic compute resources}

Figure 4 compares the cost of transferring data to compute resources between NLP-solver based source selection and single source selection given by CloudFront. We set the execution cost to zero for comparing only the transfer cost. The results show that the total data transfer cost is minimized when using NLP-solver based storage host selection for all size of data. As the size of data increases from $30 \mathrm{MB}$ to $120 \mathrm{MB}$, the benefit of transferring data using NLP compared with CloudFront increases. For the total size of $120 \mathrm{MB}$ data, using the CloudFront would cost $\$ 0.025$, whereas using NLP the cost decreases to $\$ 0.020$. The difference in cost is huge for large experiments, as analyzed later in subsection 6.2.3.

The reason for the decrease in cost is NLP-solver transfers partial data in proportion to the cost of communication, as the data transfer cost is divided among all the cheapest links. CloudFront selects the a single best source for data transfer. Transferring data using CloudFront becomes more expensive as the size of these data increases.

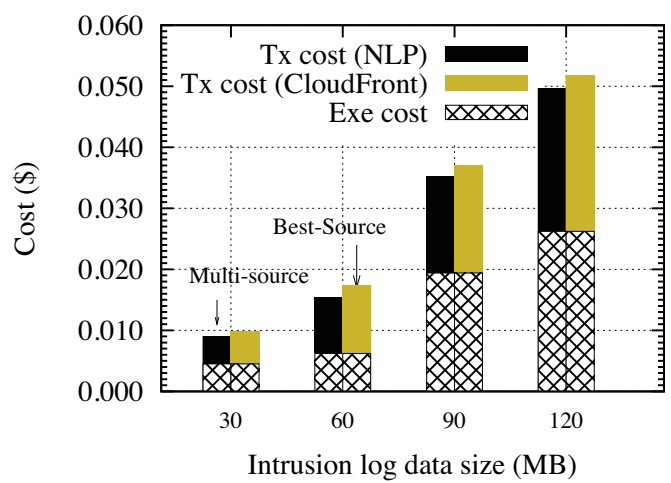

Figure 5: Comparison of total cost when both computation and transfer costs are non-zero.

\subsubsection{Scenario 2: Data and execution on Cloud re- sources}

Figure 5 depicts the total cost of executing the intrusion detection workflow on Cloud resources when using NLPsolver based task-resource mapping and (a) NLP-solver based data source selection (labelled as NLP in the figure), (b) CloudFront based data source selection (labelled as CloudFront in the figure). The NLP based task-resource mapping was used to make a fair comparison on data transfer cost between our approach and CloudFront. In this case, the NLP-model embeds the minimization of both the execution costs and data transfer costs into one objective function to be minimized, as listed in Figure 3. As two costs were involved, the total cost increased when compared to only the data transfer cost depicted in Figure 4. Nevertheless, partial data transfers based on NLP-based data source selection incurred the minimal cost for all range of data sizes.

Even when the task-resource mapping was based on NLP, the total cost savings for $120 \mathrm{MB}$ of data processed was $\$ 0.02$ on average for 1 execution. If both task-resource mapping and data retrievals were based on existing heuristics (earliest finish time for compute and best resource for data), our approach would have had more savings.

\subsubsection{Total cost savings}

Figure 6 depicts the cost of executing the real-time analysis section, depicted as Block $\mathrm{C}$ in Figure 2), 10,000 


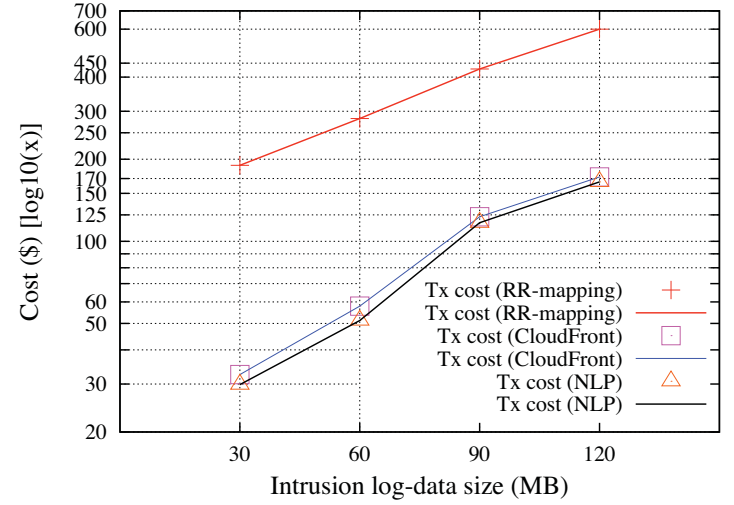

Figure 6: Comparison of total execution cost between NLP based mapping (with and without CloudFront) (round-robin based mapping as an upper-bound only).

Table 1: Distributed Compute Resources

\begin{tabular}{l|r|r||r|l}
\hline Physical Compute Nodes & cores & $\begin{array}{r}\text { Ex cost } \\
\$ / h r\end{array}$ & $\begin{array}{r}\text { DTx cost } \\
\$ / G B\end{array}$ & $\begin{array}{l}\text { Nearest } \\
\text { Region }\end{array}$ \\
\hline rotegg.dps.uibk.ac.at & 1 & $\$ 0.10$ & $\$ 0.170$ & Europe \\
aquila.dacya.ucm.es & 1 & $\$ 0.10$ & $\$ 0.170$ & Europe \\
tsukuba000.intrigger.omni.hpcc.jp & 8 & $\$ 0.80$ & $\$ 0.221$ & Japan \\
omii2.crown-grid.org & 4 & $\$ 0.40$ & $\$ 0.210$ & China \\
snowball.cs.gsu.edu & 8 & $\$ 0.80$ & $\$ 0.170$ & US \\
node00.cs.binghamton.edu & 4 & $\$ 0.40$ & $\$ 0.170$ & US \\
belle.csse.unimelb.edu.au & 4 & $\$ 0.40$ & $\$ 0.221$ & Japan \\
manjra.csse.unimelb.edu.au & 4 & $\$ 0.40$ & $\$ 0.221$ & Japan \\
\hline
\end{tabular}

times (Blocks A and B are usually computed only once for each set of data). The cost values for each data group were derived from the cost of 10 executions multiplied by 1000. The most costly approach was when using roundrobin based task-resource mapping algorithm and nearest source data retrievals. This value should be interpreted as an upper bound for comparison purposes only. This cost was reduced by $77.8 \%$ (\$466.8) when we used the NLP-solver based mapping and multi-source partial data retrieval; and by $76.2 \%$ ( $\$ 457.2)$ when we used NLP-solver based mapping and data retrieval from CloudFront's best source. This would amount to savings of three-quarters of the total expenditure if intrusion detection systems were to be executed on Clouds using our model. When the costs obtained by using NLP based approach was compared to CloudFront's, NLP was able to reduce the cost by $\$ 7.1$ on average. This cost savings would cumulate to be higher for larger data and repeated experiments. Thus, for all scenarios, the total cost incurred when using NLP-solver is lower than the cost incurred when using Amazon's CloudFront based data retrieval.

We tabulated the cost of computation and data transfer according to Amazon's current pricing policy in Table 1. The highest computation cost of Amazon Cloud resources is more than the highest data transfer cost ${ }^{6}$. Armbrust et al. [1] have compared the normalized cost of computing resources and WAN bandwidth between 2008 and 2003. Their data clearly shows that the cost/performance improvement is 2.7

\footnotetext{
${ }^{6}$ assuming transferring 1GB from Amazon takes 1 hour of CPU time
}

times and 16 times for WAN bandwidth and CPU hours, respectively. This trend hints to the fact that data transfer costs are not decreasing as much as computation cost. Hence, for data-intensive applications, total cost savings on communication is a necessity as compared to computation cost.

\section{Related Work}

Armbrust et al. [1] described the benefits of moving to Cloud computing. These benefits include lower operating costs, physical space savings, energy savings and increased availability.

Deelman et al. [5] presented a case study for examining the cost-performance tradeoffs of different workflow execution modes and provisioning plans for Cloud resources. They concluded that data-intensive applications can be executed cost-effectively using Cloud computing infrastructure. In our paper, we focus on the minimization of communication cost using globally distributed Cloud edge-servers and compute nodes.

Amazon CloudFront uses edge locations in United States, Europe, and Asia to cache copies of the content for faster delivery to end users. It provides users address in the form of a HTTP/HTTPS uniform resource locator (URL) . When a user requests one of these data from any site, Amazon CloudFront decides which edge location is 'best' able to serve the request to that user's location. However, users do not have control over the amount of data to get from each edge servers, to minimize cost, unless they access the URL from a different location. We compare our approach with this 'best' location approach.

Wu et al. [18] presented the design and implementation of Collaborative Intrusion Detection System (CIDS) for efficient intrusion detection in a distributed system. They claim that aggregate information is more accurate than elementary data for intrusion detection.

Zeller et al. [19] presented the advantages of using Cloud computing for data mining applications, especially when the size of data is huge and globally distributed.

Broberg et al. [2] introduced MetaCDN, which uses 'Storage Cloud' resources to deliver content to content creators at low cost but with high performance (in terms of throughput and response time).

Microsoft has a Windows Workflow Foundation for defining, managing and executing workflow as part of its .NET services. With the .NET services, workflows can be hosted on Clouds for users to access it from anywhere [13]. The service facilitates transparent scalability for persistence stores and distribution of load between hosts.

A number of work in Grid computing, especially those related to Data Grids, have focused on optimal selection of data sources while scheduling applications $[16,12]$. Also, some existing workflow systems $[6,8,11]$ use a variety of optimization metrics such as the execution time, effi- 
ciency, economical cost, or any user-defined QoS parameter for scheduling workflow applications. In Grids, users were not able to provision required type of resources at specified locations as demanded by applications. In Clouds, however, users can first choose the set of compute and storage resources they want for their application and then use our model for minimizing the total cost. The initial selection may be based on user's budget allocated for executing the application in Clouds.

\section{Conclusions}

In this work, we presented the execution of an intrusion detection application workflow using Cloud resources, with an objective of minimizing the total execution cost. We modeled the cost minimization problem and solved it using a non-linear program solver. Based on the solution, we retrieved data from multiple data sources to the compute resource where a task was mapped, unlike previous approaches, where data was retrieved from the 'best' data source. Using our NLP-model we achieved savings of three-quarters of the total cost as compared to using CloudFront's 'best' data source selection, when retrieving data.

We conclude that by dividing data retrievals to distributed datacenters or storage Clouds in proportion to their access cost (as in our model), users can achieve significant cost savings than when using existing techniques. As part of our future work, we would like to explore proactive scheduling of data retrievals across multiple datacenters in Clouds.

\section{Acknowledgments}

This work is partially supported through Australian Research Council (ARC) Discovery Project grant. We thank University of Tokyo, University of Innsbruck, Poznan Supercomputing Center, Universidad Complutense de Madrid, Beihang University, Georgia State University, SUNY Binghamton for providing us compute nodes for our experiments. We would also like to thank James Broberg, Anton Beloglazov and Marco Netto.

\section{References}

[1] M. Armbrust, A. Fox, R. Grifth, A. D. Joseph, R. Katz, A. Konwinski, G. Lee, D. Patterson, A. Rabkin, I. Stoica, and M. Zaharia. Above the clouds: A berkeley view of cloud computing. Technical report, University of California at Berkeley, February 2009.

[2] J. Broberg, R. Buyya, and Z. Tari. Metacdn: Harnessing 'Storage Clouds' for high performance content delivery. Journal of Network and Computer Applications, 32(5):1012-1022, 2009.

[3] R. Buyya, S. Pandey, and C. Vecchiola. Cloudbus toolkit for market-oriented cloud computing. In CloudCom '09: Proceedings of the 1st International Conference on Cloud Computing, volume 5931 of LNCS, pages 24-44. Springer, Germany, December 2009.
[4] R. Buyya, C. S. Yeo, S. Venugopal, J. Broberg, and I. Brandic. Cloud computing and emerging it platforms: Vision, hype, and reality for delivering computing as the 5th utility. Future Generation Computer Systems, 25(6):599_ 616, 2009

[5] E. Deelman, G. Singh, M. Livny, B. Berriman, and J. Good. The cost of doing science on the cloud: the montage example. In SC '08: Proc. of the 2008 ACM/IEEE conference on Supercomputing, pages 1-12, Piscataway, NJ, USA, 2008.

[6] T. Fahringer, A. Jugravu, S. Pllana, R. Prodan, C. Seragiotto, Jr., and H.-L. Truong. Askalon: a tool set for cluster and grid computing: Research articles. Concurrency and Computation: Practice \& Experience, 17(2-4):143-169, 2005.

[7] R. Fourer, D. M. Gay, and B. W. Kernighan. AMPL: A Modeling Language for Mathematical Programming. Duxbury Press, November 2002.

[8] A. Geppert, M. Kradolfer, and D. Tombros. Market-based workflow management. International Journal of Cooperative Information Systems, World Scientific Publishing Co, 7 , 1997.

[9] K. K. Gupta, B. Nath, and K. Ramamohanarao. Layered Approach using Conditional Random Fields for Intrusion Detection. IEEE Transactions on Dependable and Secure Computing. In Press.

[10] W. Lee, S. J. Stolfo, and K. W. Mok. A data mining framework for building intrusion detection models. In IEEE Symposium on Security and Privacy, pages 120-132, 1999.

[11] A. Mayer, S. McGough, N. Furmento, W. Lee, S. Newhouse, and J. Darlington. Iceni dataflow and workflow: Composition and scheduling in space and time. In UKe-Science All Hands Meeting, pages 627-634. IOP Publishing Ltd, 2003.

[12] R. McClatchey, A. Anjum, H. Stockinger, A. Ali, I. Willers, and M. Thomas. Scheduling in data intensive and network aware (diana) grid environments. Journal of Grid Computing, 5(1):43-64, 2007.

[13] Microsoft. Windows Azure Platform .NET Services. http://www.microsoft.com/azure/netservices.mspx.

[14] S. Pandey, W. Voorsluys, M. Rahman, R. Buyya, J. Dobson, and K. Chiu. A grid workflow environment for brain imaging analysis on distributed systems. Concurrency and Computation: Practice \& Experience, 21(16):2118-2139, November 2009.

[15] P. Spellucci. A sqp method for general nonlinear programs using only equality constrained subproblems. Mathematical Programming, 82:413-448, 1993.

[16] S. Venugopal, R. Buyya, and K. Ramamohanarao. A taxonomy of data grids for distributed data sharing, management, and processing. ACM Computing Surveys, 38(1), 2006.

[17] I. H. Witten and E. Frank. Data Mining: Practical Machine Learning Tools and Techniques. Morgan Kaufmann, San Francisco, 2 edition, 2005

[18] Y.-S. Wu, B. Foo, Y. Mei, and S. Bagchi. Collaborative intrusion detection system (cids): A framework for accurate and efficient ids. In Proceedings of the 19th Annual Computer Security Applications Conference (ACSAC 03). Purdue University, Applied Computer Security Associates, 2003.

[19] M. Zeller, R. Grossman, C. Lingenfelder, M. R. Berthold, E. Marcade, R. Pechter, M. Hoskins, W. Thompson, and R. Holada. Open standards and cloud computing: Kdd-2009 panel report. In $K D D$ '09: Proceedings of the 15th ACM SIGKDD international conference on Knowledge discovery and data mining, pages 11-18, NY, USA, 2009. ACM. 